\title{
RESEARCH ON MACHINE VISION BASED AGRICULTURAL AUTOMATIC GUIDANCE SYSTEMS
}

\author{
Bin Liu ${ }^{1}$, Gang Liu ${ }^{1, *}$, Xue $\mathrm{Wu}^{2}$ \\ ${ }^{1}$ Key Laboratory of Modern Precision Agriculture System Integration Research, China \\ Agricultural University, Beijing, China, 100083 \\ ${ }^{2}$ College of Mechanical Engineering and Automation, Beijing Technology and Business \\ University, Beijing, China, 100037 \\ * Corresponding author, Address: P.O. Box 125, Key Laboratory of Modern Precision \\ Agriculture System Integration Research, China Agricultural University, 17 Tsinghua East \\ Road, Beijing, 100083, P. R. China, Tel: +86-10-62736741, Fax: +86-10-62736746, Email: \\ pac@cau.edu.cn
}

Abstract: With the concept of precision agricultural was proposed, the research of agricultural automatic vehicle was paid more attention to in the world. The fundamental element of machine vision based agricultural automatic navigation system was presented in this paper. It includes path finding, location \& path tracing and motion controlling. The image processing, automatic control and sensor fusion techniques are the key for autonomous vehicle systems.

Keywords: Agricultural vehicle, Machine vision, Guidance, Automatic control

\section{INTRODUCTION}

With the research and practice of precision agriculture nowadays, new mode of agricultural production and the application of new technology promote the development of agricultural vehicle. In the 1990s, automation, information and intelligence which have been promoted rapidly by the computer and information collecting technology play the key role in vehicle automation of modern agriculture. With the rapid advancement in 
electronics, computers and computing technologies, the intelligent agricultural vehicle with autonomous navigation system has been used for many field operations such as planting, fertilizing, tillage, and harvesting.

According to the navigation methods of agricultural vehicle guidance systems, various navigation technologies include: (1) mechanical navigation (2) GPS (Global Positioning System) navigation (3) machine vision navigation (Han et al., 1990).

GPS-based guidance system is a kind of absolute navigation way which is based on some known position points from GPS receiver; in contrast, machine vision guidance is a relative position and heading method based on tracking forward directrix. Compared with GPS-based guidance, machine vision based guidance has great potential for implementation of agricultural vehicle navigation system. Real-time characteristic for detecting obstacle and high cost performance are the superiority of machine vision guidance. As a result, machine vision guidance has been an interest for agriculture researchers.

\section{KEY TECHNIQUES OF MACHINE VISION BASED AGRICULTURAL VEHICLE GUIDANCE SYSTEM}

The intelligent navigation system based on machine vision has three key elements: path finding, location \& path tracing and motion controlling, as shown in Fig. 1. Firstly, path finding is the key in achieving accurate control of the vehicle. Image information collected by image sensor from crop row structure is used for detecting navigation features and finding out the effective navigation course (directrix). Agricultural vehicle is mainly be used in nature environment, therefore image information process technology must have the features of real-timing, robustness and adaptability for nature light. Secondly, according to the result of path finding and position \& heading information from other sensors, location \& path tracing is to locate the relative position and heading of agricultural vehicle in crop rows. Path planning is then providing an offset that is used with a steering gain to directly control the wheel position. Finally, motion controller is to adjust oriented angle of front wheel according to the navigation planning information. Then agricultural vehicle can be guided in crop rows autonomously by a machine vision-based navigation system.

In a vision-based vehicle guidance system, finding guidance information (path finding) from crop row structure is the key in achieving accurate control of the vehicle operation. A number of image information processing techniques have been used in path finding. In nature environment, the fact 
that farmland sense is complicated and non-structural make image processing more difficult. So control parameters which are offered by the machine vision navigation system cannot keep up with the vehicle's status shift. The final result is that validity of navigation control will be affected.

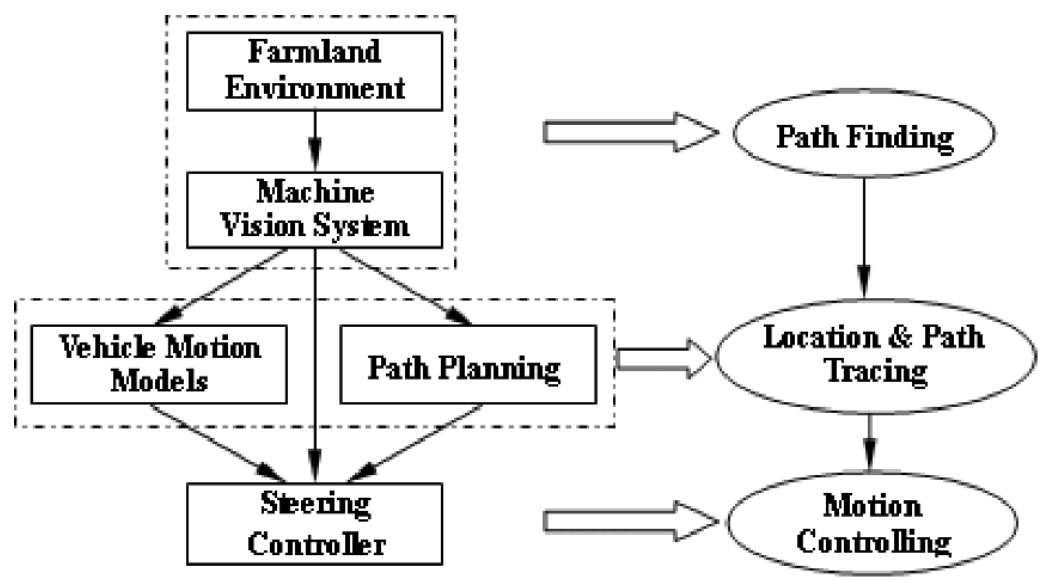

Fig. 1. Key elements of intelligent navigation system

\section{VISION-BASED IMAGE PROCESSING TECHNOLOGY}

The main target of image processing in the machine vision navigation is to perceive guidance course and to detect obstacle, further, to locate relatively. Path finding and surroundings Perceiving of the vision-based guidance system can be defined as four processing, as shown in Fig. 2.

The key of navigation system research based on machine vision is to isolate target from background, yet traditional edge-detection method is unsatisfactory in crop images processing. One of the problems encountered in outdoor image acquisition is the unevenness of the lighting conditions, in time and space. The variations of the sun elevation and the nebulosity contribute to change the global illumination of the scene observed by the camera. Another problem is that in farmland images the most outline feature of aqueduct, ridge of field and crop is lost. The edge of objects cannot be detected by using traditional processing method. For this reason, a new edgedetection method based on color features has been developed by researchers.

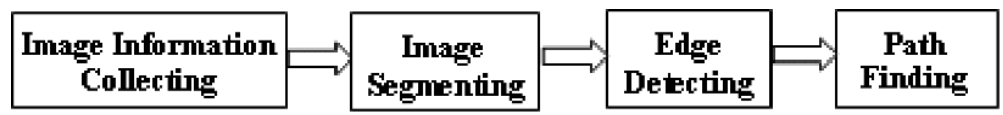

Fig. 2. Four steps of image processing in vision-based guidance 


\subsection{Color features based segmentation}

Crop images from a standard RGB sensor include the most direct information of color features. There are the obvious difference between plants and soil that plant show green and soil is brown in image. So color feature of green plant can be used in segmentation between objects and background. This processing step is also called image pretreatment, as shown in Fig. 3. Jiang zhengrong (1999) investigated weed identification by simulating farmland conditions, and the result showed that difference of (G-B)//R-Gl parameter and 2G-R-B parameter between plants and soil is obvious. Woebbeck and Meyer (1995) researched on using color feature to identify weed in different soil, crop and sunlight conditions, and finally they led that $2 \mathrm{G}-\mathrm{R}-\mathrm{B}$ is the most effective way (parameter) for distinguishing weed on soil background in crop image processing. Therefore, 2G-R-B can be regarded as the color model for distinguishing plants on soil background in colorful crop image segmentation.

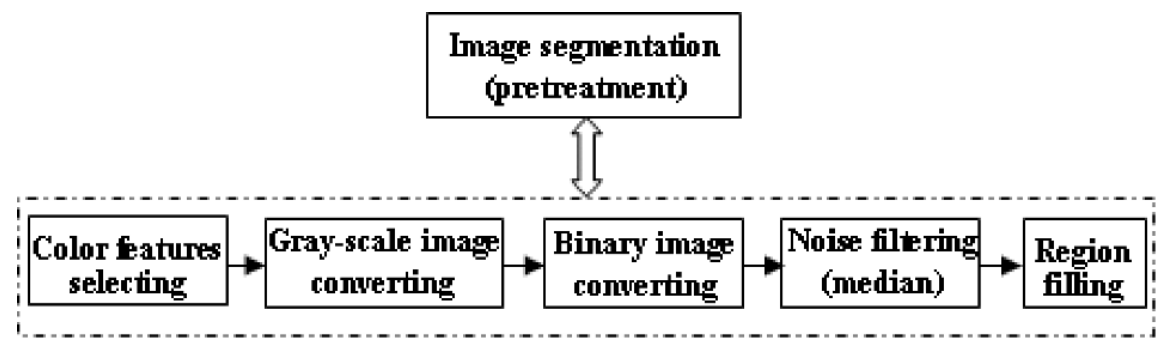

Fig. 3. Image segmentation

The first step is to manipulate the image in such a way that the living plant tissue is emphasized on Green color feature in comparison with all other objects in the image. This is done by first dividing the color image into its red, green and blue channels. Subsequently, an indicator value for living plant tissue is computed as: Indicator $=2 \times$ Green - Red - Blue.

To visualize the indicator values as a grey-scale image, they may be mapped to the range of 0-255 by simple linear mapping. This image is well suited for subsequent convert gray-scale image to binary image.

The second step is to convert gray scale image into a binary image. It is also called image thresholding. Selecting appropriate threshold is very important for separating crop pixels from the background. However, it is very difficult that conforming threshold for binary image in real-time navigation. The typical solution is to predefine segmentation threshold in initialization.

The final step is to find the boundary between objects and background. There is much noise in crop images, and it may affect the accuracy of edge 
detection algorithm. The median filter is well suited to remove spikes and thin lines from the pre-treated image. So the median filter can be applied on the final step. Moreover, there are some holes in bi-level images. Filling holes is an effective method to remove the holes.

Objective region is separated from the background after pre-treated image.

\subsection{Edge detection}

Followed by pretreatment, Morphological algorithm can be introduced to detect the edge of objective region. Edge detection based on morphology can be regard as:

$$
\beta(A)=A-(A \Theta B)
$$

Where

$\beta$ (A) is the edge of an objective region $\mathrm{A}$.

$B$ is the suitable structural element. The structure $3 \times 3$ element can be used.

\subsection{Guidance directrix extraction}

After the edge of objective region detected, guidance directrix can be gained through image processing. Usually, Hough transform is adapted. The Hough transform is widely used for localization of linear objects in images (Sonka et al., 1993).

Hough transform is a kind of linear description way in which point $(\mathrm{x}, \mathrm{y})$ in Descartes coordination system is converted into a sine curve $(\rho=x \cos \theta+y \sin \theta)$ in polar coordinate system. Subsequently, a number of points in Descartes coordination system will be converted into the corresponding curves. The intersection of these curves in a certain range can be used to find out the best fitting line. According to the transform equation, every point in image can be represented by the parameters $(\rho, \theta)$ of polar coordination system, and the maximum value of the intersections in polar coordination system indicates that a line has been found.

\section{LOCATION AND TRAJECTORY TRACKING}

Location and Trajectory tracking are important for agricultural vehicle control. Most guidance operations require that the vehicle follow some nominal trajectory or directrix. Local information can be the source of directrix and sensed directly. These include crop rows, swath edges, tilled/untilled boundaries. In parallel swathing, the directrixis parallel lanes to some prior path. The directrix might also be defined by a desired course from a map or script. Many research efforts in guidance have dealt with 
guidance in straight lanes and thus the role of the navigation planner is much simplified. For curved row guidance, the navigation planner must consider the sensor information and vehicle motion to navigate in the desired course. The navigation planner also plays an important role in autonomous operation, providing different machine 'behaviors' based on field conditions (Reid et al., 2000).

A finite state approach was developed by CMU in their autonomous windrower (Hoffman, 1996). Primary states of the machine triggered different behaviors or operations. Some basic states defined which side of the harvester was uncut crop, identified the proximity of a turn, and the implemented of turning functions. In each state of operation the machine performed one or more behaviors. For example, harvesting a rectangular field of alfalfa in a simple serpentine pattern required combinations of the following states with associated behaviors (Reid J.F. et al., 2000):
$\diamond \quad$ Cut on right
$\diamond \quad$ Near right turn
$\diamond \quad$ Turn right
$\diamond \quad$ Cut on left

\section{AGRICULTURAL VEHICLE CONTROL TECHNOLOGY}

The research on agricultural vehicle control technique is focus on designing the steering controller systems which is the main purpose. The steering controller is the actuator that converts a control signal from a feedback controller to an appropriate mechanical adjustment in steering angle. Agricultural equipment often operates on unprepared, changing and unpredictable terrain. In the case of automatic or autonomous operation, steering controllers should be able to provide appropriate steering actions in response to the variations in equipment operation states, traveling speed, tire cornering stiffness, ground conditions, and many other parameters influencing steering dynamics.

Since most modern agricultural vehicles employ some form of hydraulic steering system, various factors such as electrohydraulic $(\mathrm{E} / \mathrm{H})$ steering unit, dynamic feature of vehicle, road conditions, and vehicle speed and so on should be taken in account adequately in designing steering controller (Reid et al., 2000).

A classical model based steering controller was developed by Stombaugh et al. (1998) for high speed agricultural tractor. They found that the steering controller must compensate for the dynamics of both the vehicle and the steering system when these two systems were in the same frequency range. 
Benson et al. (1998) designed a PID steering controller in frequency domain for an agricultural tractor guided by a GDS. Its closed loop transfer function was obtained experimentally. Test results showed that steering controller played an important rule in achieving satisfactory automated guidance of an agricultural tractor.

Wu et al. (1998) developed a methodology for designing E/H steering PID controllers for agricultural vehicles. In their approach, a linearized dynamic model of the steering system was used to design the controller.

In the research on controlling system, many problems need to be solved. Those problems include how to modify electrohydraulic controller, how to keep up the stability of electrohydraulic control system, how to deal with sideslip of vehicle's turning in high speed, how to realize real-time control, how to solve deadband and nonlinear question of operation system, how to control automatically agriculture vehicle in upland.

\section{CONCLUSIONS}

In study of automatic navigation system of agriculture based on machinevision, there still have several questions to be solved as followed.

Firstly, the real-time characteristic of image processing method should be fulfilled. When sense image collected in nature environment become more and more complicate, the time for processing image will increase greatly. If the speed of image process is even low and nonstructural environment is unknown or changeable, not only the traditional detecting-modelingplanning way but also detecting-action control way will not realize effective control.

Secondly, multi-sensors-based information fusing technique will play an important role in intelligent guidance system. Although machine vision navigation has high flexibility, its navigating accuracy is affected by environment and surroundings features. Through fusing variety of information from GPS and other sensors, accuracy and dependability of navigation will be advanced. Furthermore, multi-sensors-based information fusing technique can solve special problems such as without effective surroundings feature for turning around in the end of farmland.

Finally, effective control algorithm is crucial. In the complicate environment, there has nonlinear and random disturbance in moving vehicle. Because intelligent controlling method doesn't rely on mathematical models, it has good controlling effect and robustness. In the environment which can be known or predicted, investigators can establish effective fuzzy ruler by experiment, simulation and expert experience. Yet persons have no way to predict all the conditions in complicated environment, controlling system of guidance system should have the capability of self-learning and self-adapting. 


\section{ACKNOWLEDGEMENTS}

This paper is supported by the national 863 projects: Control Technique and Product Development of Intelligent Navigation of Farming Machines (2006AA10A304).

\section{REFERENCES}

Benson, E. R., Reid, J. F., Zhang, Q., Machine-vision based guidance system for agricultural grain harvester using cutedge detection [J]. Biosystem Engineering, 2003, 86(4): 389-398.

Benson, E., Stombaugh, T., Noguchi, N., Will, J., Reid, J.F., 1998. An evaluation of a geomagnetic direction sensor for vehicle guidance in precision agriculture applications. ASAE Paper 983203. ASAE. St. Joseph, MI.

Han, S., Zhang, Q., Reid, J.F., A guidance directrix approach to vision-based vehicle guidance systems [J]. Computers and Electronics in Agriculture, 2004, 43: 179-195.

Jiang Zhengrong, Implementation and Application of Computer Technique for Identification of Weed [J]. Weed science, 1999, 4: 2-5. (in Chinese)

Reid, J.F., Zhang, Q., Noguchi, N., Agricultural automatic guidance research in North America [J]. Computers and Electronics in Agriculture, 2000, 25: 155-167.

Søgaard, H.T., Olsen, H.J., Determination of crop rows by image analysis without segmentation [J]. Computers and Electronics in Agriculture, 2003, 38: 141-158.

Stombaugh, T., Benson, E., Hummel, J.W., 1998. Automatic guidance of agricultural vehicles at high field speeds. ASAE Paper 983110. St. Joseph, MI.

Sun Yuanyi, Zhang Shaolei, Li Wei, Guidance lane detection for pesticide spraying robot in cotton fields [J]. Journal of Tsinghua University (Science \& Technology), 2007, 47(2): 206-209. (in Chinese)

Tian Haiqing, Ying Yibin, Zhang Fangming, Development of Automatic Control Technique for Agricultural Vehicle Guidance [J]. Transactions of The Chinese Society of Agricultural Machinery, 2005, 36(7): 148-152. (in Chinese)

Leemans, V., Destain, M.-F., Application of the Hough Transform for Seed Row Localisation using Machine Vision [J]. Biosystems Engineering, 2006, 94(3): 325-336.

Woebbecke, D.M., Meyer, G.E., Von Bargen, K. et al. Color indices for weed identification under various soil, residue, and lighting conditions [J]. Trans of the ASAE, 1995, 38(1): 259-269.

Wu, D., Zhang, Q., Reid. J.F., Qiu, H., Benson, E.R., 1998. Model Recognition and Simulation of an E/H Steering Controller on Off-Road Equipment. In: Nair, S.S., Mistry, S.I. (Eds). Fluid Power Systems and Technology 1998, ASME, New York, pp. 55-60.

Zhang Fangming, Ying Yibin, Review of Machine Vision Research in Agricultural Vehicle Guidance [J]. Transactions of The Chinese Society of Agricultural Machinery, 2005, 36(5): 133-136. (in Chinese) 